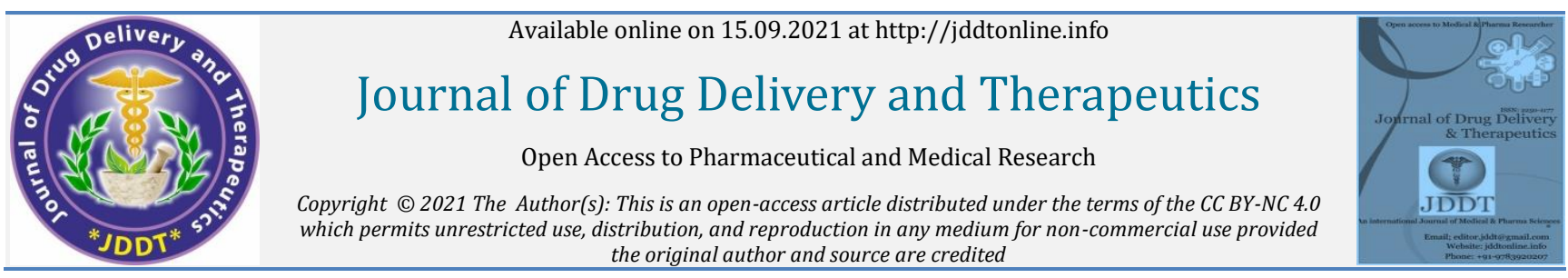
the original author and source are credited

Review Article

Open Access Full Text Article

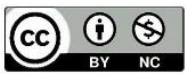

\title{
A Review on Niosomes: Potential Vesicular Drug Delivery System
}

\author{
Kumavat Sonali, Sharma Pravin Kumar*, Koka Sweta S., Sharma Ravi, Gupta Ashish, Darwhekar G. N. \\ Acropolis Institute of Pharmaceutical Education and Research, Indore (M.P), India. 453771
}

Article Info:

$\begin{array}{ll} & \text { Article History: } \\ & \text { Received 12 July 2021 } \\ & \text { Accepted 06 Sep 2021 } \\ & \text { Published 15 Sep 2021 }\end{array}$

Cite this article as:

Kumavat S, Sharma PK, Koka SS, Sharma R, Gupta A Darwhekar GN, A Review on Niosomes: Potential Vesicular Drug Delivery System, Journal of Drug Delivery and Therapeutics. 2021; 11(5):208-212

DOI: http://dx.doi.org/10.22270/jddt.v11i5.5046

\section{Abstract}

Over the years researchers have been making an attempt to improve the potency of utilization of medicament for treatment of numerous diseases. During this endeavour, drug delivery systems have helped greatly by achieving reduced dose, reduced dosage frequency, improved patient compliance, improved bioavailability and maximum concentration of the drug at the target site. Recent years have seen an unprecedented growth in the use of nanotechnology in designing drug delivery systems. Niosomes are vesicles composed of nonionic surfactants, which are biodegradable, comparatively non-toxic, stable, economical and effective alternate to liposomes. This review is deepening and widening the interest of nNiosomes in several scientific disciplines and notably its application in drug delivery. This review additionally presents a summary of the types, methods of preparation, characterization and applications of Niosomes.

Keywords: Niosomes, types, method of preparation, factors affecting and applications

*Address for Correspondence:

Sharma Pravin Kumar, Acropolis Institute of Pharmaceutical Education and Research, Indore (M.P), India. 453771

\section{INTRODUCTION}

The objective of targeting drug delivery systems is to deliver the drug in the body in such a way that it should show its action to the targeted and desired site to reach the therapeutic response, i.e. wherever its action should be required by limiting undesirable interaction to non-targeted tissues. Paul Ehrlich introduced this idea in 1909 and he referred to as this strategy "magic bullets."1 During the past decade, design of vesicles as a tool to boost drug delivery has created lots of interest amongst the scientist working in the field of drug delivery systems. Vesicular system like liposomes, niosomes, transferosomes, pharmacosomes and ethosomes provide alternate to improve the drug delivery. ${ }^{2}$

Among these different carriers niosomes are well effective drug delivery systems. ${ }^{3}$ Niosomes are vesicular, novel drug delivery system, which may be used for the sustained, controlled as well as targeted delivery of medication with high stability. ${ }^{4}$ Liposomes were the primary vesicular drug delivery systems however it shows many disadvantages like their toxicity and stability problems at completely different $\mathrm{pH}$. Due to the drawbacks of liposomes, analysis interest shifted towards niosomes.

Niosomes are unilamellar, oligolamellar or multilamellar and non-toxic because of these non ionic surfactants. Additionally they contain cholesterol or its derivatives and charged molecules. Cholesterol provides rigidity to the vesicle and the charged molecule keeps the preparation stable. $^{5}$ Surfactant-based niosomes are biodegradable, biocompatible and non-immunogenic. They act as a drug depot within the body, whereby they release medication in a very controlled manner through their closed bilayer structure, leading to a sustained release of the bounded drug to the target site. The therapeutic effects of medication enclosed in niosomes are improved by reduced clearance and specific targeting. Owing to their deliquescent, amphiphilic and lipophilic nature, niosomes are ready to accommodate a different type of medication with a varied solubility. ${ }^{6}$

The medication is encapsulated in a vesicle which are very small, and nanoscopic in size (ranges between 10 to 1000 $\mathrm{nm}) .{ }^{7}$ Numerous strategies of administration of niosomal formulation embrace intravenous intramuscular, peroral and transdermal. ${ }^{8}$ Bioavailability of poorly soluble medication is also improved and therefore the effectiveness of topical applications can be increased with the utilization of niosomes. Moreover, labile and sensitive medication is also delivered with greater ease as niosomes protect the encapsulated active pharmaceutical ingredients from deleterious conditions both inside and outside of the body. 6,9

\section{COMPONENTS OF NIOSOMES:}

\section{Non-ionic surfactants}

Non-ionic surfactants are one of the most important components of niosomes. Different kinds and their combinations are used to entrap various medications to form niosomes. The non-ionic surfactants are amphiphilic, 
biodegradable, biocompatible and non- immunogenic in nature. The characteristics of formulated niosomes are based on composition, additives concentration, size, lamellarity and surface charge of vesicles. The following types of non-ionic surfactants are utilized for the formation of niosomes eg. Span $(60,40,20,85$, and 80$)$ and Tween $(20$, $40,60,80) \cdot{ }^{10}$

\section{Cholesterol}

It is a very important additive for formulation of niosomes. Cholesterol is not only required for the formulation of niosomes but its presence affects many properties of the niosomes. It has an effect on permeability of the membrane, rigidity of the membrane, entrapment efficiency, ease of rehydration of freeze-dried niosomes, stability and storage time. If the cholesterol is employed with low HLB surfactants, it increases the stability of the vesicle and if the HLB value is more than 6 , it helps in the formation of bilayer vesicles. The addition of cholesterol increases the viscosity and hence rigidity of the formulation 5 .

\section{Charged molecule}

Some charged molecules are added to niosomes to extend stability by providing electric repulsion to avoid collision. The negatively charged molecules are used dicetyl phosphate (DCP) and phosphotidic acid. Similarly, stearyl amine and stearyl pyridinium chloride are renowned charged molecules utilized in niosomal preparations. ${ }^{11,12}$

\section{Hydration medium}

Hydration medium is one of the most important components required in the formulation of niosomes. Generally, phosphate buffer is used as a hydration medium. But the $\mathrm{pH}$ of the buffer depends on the encapsulated drug solubility.12

\section{ADVANTAGES OF NIOSOMES}

- Niosomes are less toxic and compatible since they do not carry any charge.

- These are biodegradable and do not initiate immunogenic reactions.

- It can enhance the bioavailability of the active pharmaceutical ingredient by increasing the physical as well as biological stability. ${ }^{6}$

- Handling and storage of surfactants requires no special conditions. ${ }^{13}$

- Their surface formation and modification are very easy. ${ }^{14}$

- It can improve the therapeutic performance of the medication by protecting it from the biological environment and restricting effects to target cells, thereby reducing the clearance of the drug.

- Can enhance the skin penetration of drugs.

- They increase the stability of the entrapped drug.

- The vesicles can act as a depot to release the medication slowly and provide a controlled release. ${ }^{15}$

- As vesicle suspension is water based vehicle hence provide higher patient compliance than oil based dosage forms. 3,16

\section{DISADVANTAGES OF NIOSOMES}

- Time consuming method

- Specialized equipment are needed for processing
- Aggregation

- Fusion

- Leaking of entrapped drug

- Chemical reaction of encapsulated medicine. ${ }^{5}$

\section{TYPE OF NIOSOMES}

\section{Proniosomes}

These are formed from the mixture of carrier and surfactant. And on the hydration of proniosome mixture, niosomes are produced.

\section{Aspasomes}

It is made using the mixture of ascorbyl palmitate, cholesterol and exceptionally charged lipid dicetyl phosphate prompts the arrangement of vesicles. Aspasomes are initially hydrated with water/fluid arrangement and then it is subjected to sonication to get the niosomes. Aspasomes can be utilized to build the transdermal saturation of medication.

\section{Niosomes in carbopol gel}

Niosomes are incorporated in carbopol-934 gel $(1 \% \mathrm{w} / \mathrm{w})$ base containing propanediol $(10 \% \mathrm{w} / \mathrm{w})$ and glycerine $(30 \%$ $\mathrm{w} / \mathrm{w})$.

\section{Deformable niosomes}

The mixture of non-ionic surfactants, alcohol and water form the deformable niosomes. These are smaller vesicles and simply cross the pores of stratum corneum that leads to extend penetration potency. It is employed in topical

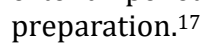

\section{METHODS OF NIOSOME PREPARATION}

\section{Thin film hydration technique}

Thin film hydration method also known as hand shaking method is a simple technique though, it requires the use of organic solvents to dissolve surfactant and cholesterol. The surfactants and cholesterol are dissolved in a round bottomed flask followed by evaporation of the organic solvent to create a thin film on the bottom of the flask. Adding of hydration medium film swells from the wall of the round bottom flask at a temperature above the transition temperature of the surfactant for a specified time period with constant slight agitation to form vesicles, which are then treated to produce unilamellar vesicles. ${ }^{18}$

\section{Ether injection method}

The method essentially based on slowly introducing a solution of surfactant dissolved in diethyl ether into warm water on magnetic stirrer with hot plate and maintained at $60^{\circ} \mathrm{C}$. The surfactant mixture in ether is slowly injected through 14-gauge needle into a solution of drug. Vaporization of solvent leads to formation of single layered vesicles. The particle size of the niosomes vesicle varies from 50 to $1000 \mathrm{~nm}$.

\section{Sonication techniques}

In this technique, an aliquot of drug solution in buffer is transferred to the surfactant/cholesterol mixture in a glass vial. The mixture is sonicated by using probe sonicator at $60^{\circ} \mathrm{C}$ for 3 minutes titanium probe is used to yield the niosomes. 


\section{Reverse phase evaporation technique (REV)}

In this method, the surfactant and cholesterol are dissolved in a mixture of ether and chloroform. An aqueous phase containing drug is added to this and the resulting two phases are sonicated at $4-5^{\circ} \mathrm{C}$. A clear transparent gel is formed. Then it is further sonicated after addition of hydration medium. The above organic phase is removed at $40^{\circ} \mathrm{C}$. The resulting niosome suspension is diluted with phosphate buffer saline and heated at $60^{\circ} \mathrm{C}$ for $10 \mathrm{~min}$ to form niosomes. ${ }^{19}$

\section{Micro fluidization method}

In this technique, the two fluidized streams (one containing drug and the other surfactant) interact at ultrahigh velocity, in precisely defined micro channels within the interaction chamber in such a way that the energy supplied to the system remains in the area of niosomes formation. This is called submerged jet principle. It results in better uniformity, smaller size and reproducibility in the formulation of niosomes.

\section{Extrusion method}

In this technique, the mixture of cholesterol and dicetyl phosphate is prepared and the solvent of the mixture is evaporated using rotary vacuum evaporator to form a thin film. The film is then hydrated with the drug solution and the resulting suspension is obtained to extruded through the polycarbonate membrane and then placed in sequence up to eight passages to get uniform size niosomes. ${ }^{2}$

\section{Transmembrane $\mathrm{pH}$ gradient drug uptake}

The mixture of surfactant and cholesterol are dissolved in chloroform in a round bottomed flask. The solvent evaporation is done under reduced pressure to form the thin film on the wall of the flask. The film on the wall is hydrated with citric acid ( $\mathrm{pH} 4)$ by vortex mixing to form the multilamellar vesicles. Then they are freeze and thawed three times and sonicated to obtain the niosomes. ${ }^{8}$

\section{Multiple membrane extrusion technique:}

The mixture of cholesterol, surfactant and dicetyl phosphate in chloroform to form a thin film by evaporation. The thin film is hydrated by a drug media using polycarbonate membranes and it is a good technique for controlling niosome size and the final suspension is extruded through which are placed in series for up to 8 passages. ${ }^{20}$

\section{The single pass technique}

The solution or suspension of lipids are extruded with air mass through a porous device and a nozzle followed by blending to create niosomes of narrow range 50-500 nm size distribution. ${ }^{21}$

\section{FACTORS INFLUENCING THE PREPARATION OF NIOSOMES}

\section{Cholesterol}

The cholesterol in niosomes rise its hydrodynamic diameter and entrapment efficiency. It affects the membrane characteristics of the niosomes like it affects the properties of the biological membrane. It decreases the flexibility of the membrane and also affects the permeation of the drug across it. The quantity of cholesterol to be used depends on the HLB value of the surfactant.

\section{Nature of surfactant}

The ether types of surfactant with single alkyl hydrophobic tail is more harmful than the dialkyl ether chain. The ester based surfactants are chemically less stable than ether kind surfactant as ester linkage is extra prone for degradation by esterase to fatty acids and triglycerides. Rise in the HLB value of surfactants leads to improve the mean size of niosomes due to the reduction in surface free energy with rise in the surfactant hydrophobicity.

\section{Structure of surfactants}

This composition, which might be connected to essential loading variables, influences the form of the vesicle to be made from surfactants. Supported vital chemical agent packaging variables, vesicle geometry is expected to be developed. ${ }^{22}$

\section{Hydration temperature}

The niosomes size and shape is affected by the hydration temperature. Temperature of hydration should be above the gel liquid phase transition temperature. Variation in temperature affects the assembly of surfactants into vesicles and vesicle shape modification. The volume and time of hydration medium also accounts for the modification. Unsuitable hydration time, temperature and hydration volume which may raise problem in niosome formation like yields the fragile niosomes or cause drug leakage.

\section{Hydration time}

Niosomes of methylene blue is prepared using thin film hydration technique and probe sonicated the vesicles for size uniformity. The effect of hydration time and hydration volume on entrapment efficiency and vesicle size is studied. It suggested that short hydration time produces vesicles with larger sizes and less drug entrapment and longer hydration time produces small-sized vesicles. ${ }^{5}$

\section{Charge}

Charge of the vesicle improve the inter lamellar distance between successive bilayers in multilamellar vesicle structure and it also improve the overall entrapped volume and stability of niosomes. ${ }^{8}$

\section{Resistance to osmotic stress}

Addition of a hypertonic salt solution to a suspension of niosomes brings about decrease in diameter. In hypotonic salt solution, there is initial slow release with slight swelling of vesicles probably due to inhibition of eluting fluid from vesicles, followed by quicker release, which may be due to mechanical loosening of vesicles structure below osmotic stress. $^{20}$

\section{Nature of encapsulated drug}

Niosomal formulation is influenced by the nature of the drug being encapsulated. The interaction of the surfactant head groups lead to entrapment of drug in vesicles and cause rise in charge. The formation of charge creates mutual repulsion of the surfactant bilayer and therefore will rises vesicle size. The HLB of the drug similarly affects the degree of entrapment.

\section{Composition of the membrane}

Stabilization of niosomes can be achieved by adding totally different additives to the surfactant mixture. The main drawback of niosomes formulation is the leakage of drug from the vesicles which might be controlled by the addition of cholesterol. Cholesterol confers higher rigidity to the membrane and therefore leakage of the drug is reduced. ${ }^{23}$ 


\section{CHARACTERIZATION OF NIOSOMES}

\section{Bilayer rigidity and homogeneity}

The bio-distribution and biodegradation of niosomes are influenced by rigidity of the bilayer. Homogeneity occurs both inside niosomes structure and between niosomes in dispersion and it can be determined by $\mathrm{p}-\mathrm{NMR}$, differential scanning calorimetry and fourier transform infrared spectroscopy method.

\section{Size and shape}

Numerous methods are used for the determination of mean diameter like as laser light scattering technique besides it is additionally determined by electron microscopy, molecular sieve chromatography, photon correlation microscopy and optical microscopy. ${ }^{24}$

\section{Stability}

The stability of niosomes is often evaluated by determining mean vesicle size, size distribution and entrapment efficiency over many month storage periods at totally different temperatures. Throughout storage, the niosomes are sampled at regular intervals and the proportion of drug which is retained into the niosomes is analyzed by UV spectroscopy or HPLC technique. ${ }^{4}$

\section{Bilayer formation}

Niosomes are designed by association of surfactants to make bilayer vesicles, which is characterized by X-cross formation by light polarization microscopy.

\section{Vesicle charge}

The surface charge plays an important role in stability of niosomes, generally the charged niosomes are extra stable as compared with uncharged vesicles. It is determined by using electophoresis method or zeta sizer. ${ }^{25}$

\section{Vesicle diameter}

Vesicle size/diameter is measured by using optical microscope with a calibrated eyepiece micrometre. The vesicle size of niosomes is measured separately for all batches and its mean value is calculated. ${ }^{26}$

\section{Entrapment efficiency}

This is calculated by determining the difference between the unentrapped and total amounts of drug. Unentrapped drug is determined by various methods such as exhaustive dialysis, gel filtration and centrifugation. Total quantity of drug can be determined by analyzing with a suitable analytical technique. Percent entrapment can then be calculated in the usual way. 27

Entrapment efficiency (\% EF) = (Amount of drug entrapped $/$ Total amount of drug) x 100

\section{In-vitro \% drug release}

In -vitro \% drug release of niosomes is often characterized by the following subsequent methods:

\section{Dialysis}

It is carried out by putting a niosomal dispersion in dialysis bag that is pre-washed and pre-soaked and are tied at both the ends. The dialysis bag is suspended into a dissolution media maintained at $37^{\circ} \mathrm{C}$ with continuous stirring. Samples are withdrawn at regular time interval and replaced with fresh sample. The withdrawn samples are analyzed for the drug content.

\section{Franz diffusion cell}

This cell assembly consists of donor and receptor compartment which is separated by cellophane membrane or dialysis membrane. Niosomal dispersion is placed at donor compartment and dissolution media i.e. phosphate buffer is placed in receptor compartment maintained at $37^{\circ} \mathrm{C}$ with continuous stirring by magnetic stirrer. The samples are taken out at suitable time intervals, replaced with fresh media and analyzed for drug content. ${ }^{28}$

\section{APPLICATIONS}

Delivery of anti-neoplastic drugs- Most anti-neoplastic drugs cause severe side effects. Niosomes can prolong the circulation and half-life of the drug by avoid the metabolism, thus reducing the side effects of the drugs. There is declined rate of proliferation of tumour and better plasma levels accompanied by slower elimination.

Transdermal delivery of drugs by niosomesTransdermal penetration of drugs are improved when they are incorporated in the niosomes and thus it helps in improving the bioavalability of many drugs. ${ }^{14}$

Niosomes as drug carriers- Niosomes can be used as drug delivery carriers for many categories of drugs as well as some diagnostic agents such as Iobitridol, used for X-ray imaging.

Treatment of Leishmaniasis- Leishmaniasis is a disease in which a parasite of the Leishmania attacks the cells of the liver and spleen. Niosomal formulation shows that it has potential to administer higher levels of the drug without triggering of the side effects, permits better efficacy in the treatment.

Delivery of peptide drugs- Use of niosomes enhances the stability of peptides by avoiding gastrointestinal peptide degradation. Study conducted on oral delivery of proteins and peptide drug in niosomal formulation showed improved stability of the drug.

\section{Other applications-}

Sustained release - Niosomal formulation of drug having a low therapeutic index and low solubility can provide sustained and controlled release and action.

Localized drug action - Because of nanometric size range, it also provide the localized drug action by achieving improved permeability through the epithelium tissue and connective tissue at the site of administration. ${ }^{24}$

\section{CONCLUSION}

Drug encapsulation in the niosomes provide various advantages such as improved bioavailability, sustained release, controlled release, long circulation time, reduced dosage regimen, site specificity and targeted delivery. Niosomal formulations can be administered through the various routes like oral, topical/transdermal, parenteral and ocular for achieving both systemic and local effects. Moreover, niosomes are more stable and cost effective alternate to the other vesicular carriers. Based on the above advantages of niosomes, we can conclude that it is a very promising vesicular drug delivery system which can improve the overall therapeutic performance of drugs.

\section{REFERENCES}

1. Bhavani GD, Lakshmi PV, "Recent advances of non-ionic surfactant-based nano-vesicles (niosomes and proniosomes): a brief review of these in enhancing transdermal delivery of drug" 
FJPS, 2020; 6(1):1-18. https://doi.org/10.1186/s43094-02000117-y

2. Rajera R, Nagpal K, Singh S, Mishra D, "Niosomes: a controlled and novel drug delivery system" BPB, 2011; 34(7):945-953 https://doi.org/10.1248/bpb.34.945

3. Khan R, Irchhaiya R, "Niosomes: a potential tool for novel drug delivery" JPI, 2016; 46(13):195-204 https://doi.org/10.1007/s40005-016-0249-9

4. Ag Seleci D, Seleci M, Walter J, Stahl F, Scheper T, "Niosomes as nanoparticular drug carriers: fundamentals and recent applications" JN, 2016; 1-13 https://doi.org/10.1155/2016/7372306

5. Bhardwaj P, Tripathi P, Gupta R, Pandey S, "Niosomes: a review on niosomal research in the last decade" JDDST, 2020; 56:101581. https://doi.org/10.1016/j.jddst.2020.101581

6. Yeo P, Lim C, Chye S, Kiong Ling A, Koh R, "Niosomes: a review of their structure, properties, methods of preparation, and medical applications" AB, 2017;11(4):301-314. https://doi.org/10.1515/abm-2018-0002

7. Yadav S, Mishra M, Verma G, Nayak K, Tiwari A, Shukla A, "Niosomes: an approach towards targeted drug delivery system" IJIPLS, 2016; 6(6):39- 52.

8. Vadlamudi HC, Sevukarajan M, "Niosomal drug delivery- a review" IAJPR, 2012; 2(9).

9. Kuotsu K, Karim K, Mandal A, Biswas N, Guha A, Chatterjee S, "Niosome: a future of targeted drug delivery systems" JAPTR, 2010; 1(4): 374. https://doi.org/10.4103/0110-5558.76435

10. Usman M, Ramdas K, Baviskar K, "Pesticides: an overeview." JDDT, 2017; 7(4):1-5. https://doi.org/10.22270/jddt.v7i4.1462

11. Rao N, Chowdary P, Divya Y, Laksmi T, Latha K, Sirisha P, "Niosomes: a vesicular drug delivery system" RJPT, 2018; 11(8):3731- 3736. https://doi.org/10.5958/0974360X.2018.00684.4

12. Kamal M, Maher M, Ibrahim A, Louis D, "An overview on niosomes: a drug nanocarrier" DDIPIJ, 2018; 1(5):1-9.

https://doi.org/10.32474/DDIPIJ.2018.01.000125

13. Mujoriya R, Dhamandeb K, Bodlac D, "Niosomal drug delivery system - a review" IJAP, 2011; 3(3):7-10.

14. Ahmad S, Teotia D, Kumar K, "Niosomes - a promising carrier for drug delivery" IJRDPLS, 2018; 7(4):3015-3021. https://doi.org/10.21276/IJRDPL.2278-0238.2018.7(4).30153021
15. Rai A, Alam G, Singh A, Verma N, "Niosomes: an approach to current drug delivery-a review" IJAP, 2017; 06(02):41-48.

16. Moghassemi S, Hadjizadeh A, "Nano-niosomes as nanoscale drug delivery systems: an illustrated review" JCR, 2014; 185:22-36. https://doi.org/10.1016/j.jconrel.2014.04.015

17. Sanklecha V, Pande V, Pawar S, Pagar O, Jadhav A, "Review on niosomes" APP, 2018; 3(2):1-7.

18. Chen S, Hanning S, Falconer J, Locke M, Wen J "Recent advances in non-ionic surfactant vesicles (niosomes): fabrication, characterization, pharmaceutical and cosmetic applications" EJPB, 2019; 144:18-39. https://doi.org/10.1016/j.ejpb.2019.08.015

19. Sharma D, Ali A, Aate J, "Niosomes as novel drug delivery system: review article" $\mathrm{P}, 2018 ; 6(3): 58$. https://doi.org/10.29161/PT.v6.i3.2018.58

20. Yadav J, Kulkarni P, Vaidya K, Shelke G, "Niosome: a review" JPR, $2011 ; 4(3): 632-636$

21. Sraswathi TS, Mothilal M, Jaganathan MK, "Niosomes an emerging formulation tool for drug delivery-a review" IJAP, 2019; 11(2):7-15. https://doi.org/10.22159/ijap.2019v11i2.30534

22. Khalifa F, Khalifa A, Jeganath S, Nitish B, "Niosomes as target drug delivery system: a review" IJRPS, 2020; 11(3):3198-3203. https://doi.org/10.26452/ijrps.v11i3.2435

23. Patel H, .Patel U, "Review on niosomes - a novel approach for drug targeting" IJAP, 2019; 08(01):1-6.

24. Kaur D, Kumar S, "Niosomes: present scenario and future aspects" JDDT, 2018; 8(5):35-43. https://doi.org/10.22270/jddt.v8i5.1886

25. Khanam N, Alam M, Sachan A, Gangwar S, Sharma R, "Recent trends in drug delivery by niosomes: a review" AJPRD, 2013; 1(3):115-122.

26. Mishra N, Srivastava V, Kaushik A, Chauhan, V, Srivastava G, "Formulation and in-vitro evaluation of niosomes of Aceclofenac" JSIR, 2014; 3(3):337-341.

27. Kumar G, Rajeshwarrao P, "Nonionic surfactant vesicular systems for effective drug delivery - an overview" APSB, 2011; 1(4):208-219. https://doi.org/10.1016/j.apsb.2011.09.002

28. Sudheer P, Kaushik K, "Review on niosomes - a novel approach for drug targeting" JPR, 2015; 14(1):20-25. https://doi.org/10.18579/jpcrkc/2015/14/1/78376 